\title{
Mineralization-inducing potentials of calcium silicate- based pulp capping materials in human dental pulp cells
}

\author{
Sohee Kang \\ Department of Dentistry, Yeungnam University Hospital, Daegu, Korea
}

Received: April 6, 2020

Revised: May 4, 2020

Accepted: May 7, 2020

Corresponding author:

Sohee Kang

Department of Dentistry, Yeungnam

University Hospital, 170

Hyeonchung-ro, Namgu, Daegu

42415, Korea

Tel: +82-53-620-3282

Fax: +82-53-629-1772

E-mail:dr.ssoy@gmail.com

\begin{abstract}
Background: This study was performed to provide a long-term bacterial seal through the formation of reparative dentin bridge, calcium silicate-based pulp capping materials have been used at sites of pulpal exposure. The aim of this study was to evaluate the mineralization-inducing potentials of calcium silicate-based pulp capping materials (ProRoot MTA [PR], Biodentine [BD], and TheraCal LC [TC]) in human dental pulp cells (HDPCs).

Methods: Specimens of test materials were placed in deionized water for various incubation times to measure the $\mathrm{pH}$ variation and the concentration of calcium released. The morphology of HDPCs cultured on the specimens was examined using a confocal laser scanning microscope (CLSM). Alizarin red S staining and alkaline phosphatase assays were used to evaluate mineralization-inducing potentials of the capping materials.

Results: BD showed the highest calcium release in all test periods, followed by PR and TC. $(p<0.05)$. All experimental groups showed high alkalinity after 1 day, except at 14 days. BD showed the highest cell viability compared with PR and TC after 1 and 3 days, while TC showed the lowest value $(p<0.05)$. The CLSM analysis showed that cells were well adhered and expressed actin filaments for all pulp capping materials. Mineralization by PR and BD groups was higher than that by TC group based on alizarin red S staining. BD showed significantly higher alkaline phosphatase activity than PR and TC, while TC showed the lowest value $(p<0.05)$.

Conclusion: Within the limitations of the in vitro study, BD had higher mineralization-inducing potential than PR and TC.
\end{abstract}

Keywords: Biocompatibility testing; Dental material; Dental pulp capping; Dentin

\section{Introduction}

At sites of pulpal exposure, dentinal continuity may be restored through the formation of a dentin bridge across the exposure. There have been reports regarding dentin bridging after pulp capping with agents such as calcium hydroxide. Calcium hydroxide is a preferable material to manage pulp exposure because of its antibacterial characteristic and ability to induce reparative dentin formation [1]. However, it has been reported that calcium hydroxide dissolves over time, causing tunnel defects in dentin bridges, and these bridges allow communication between the pulp and capping material [2].

These findings highlight the need to use materials capable of providing a long-term bacterial seal over capped pulps. Among them, mineral trioxide aggregate (MTA) has a better capping effect by stimulating the formation of the perfect reparative dentin bridge without any toxic chemical effects. Previous studies showed that MTA was more effective than calcium hydroxide in

Copyright (C) 2020 Yeungnam University College of Medicine

This is an Open Access article distributed under the terms of the Creative Commons Attribution Non-Commercial License (http://creativecommons.org/licenses/by-nc/4.0/) which permits unrestricted non-commercial use, distribution, and reproduction in any medium, provided the original work is properly cited. 
long-term vital pulp therapy $[3,4]$. However, high prices, difficulties in handling and application, and longer binding duration remain disadvantages of MTA. Long setting times are an obstacle to using MTA as a pulp capping material since MTA needs to be layered with other materials while it is still fresh [5].

By adding accelerators and modifiers, several researchers attempted to decrease the setting reaction time and increase the effectiveness of MTA for direct pulp capping. One calcium silicate-based pulp capping material is Biodentine (BD; Septodont, Saint-Maur-des-Fosses, France), which has the advantage of a shorter setting time of 12 minutes. BD is a powder consisting of tricalcium silicate, dicalcium silicate, calcium carbonate, calcium chloride, and zirconium oxide as a radiopacifier. Previous studies showed that BD works similarly to MTA in both in vitro and in vivo. In addition, $\mathrm{BD}$ has a positive effect on pulp cells and helps them form reparative dentin $[6,7]$. However, research data on the mineral-inducing potential of $\mathrm{BD}$ is still lacking.

TheraCal LC (TC; Bisco Inc., Schaumburg, IL, USA) is a lightcured, resin-modified calcium silicate-based material designed for use in direct and indirect pulp capping and as a protective liner under various filling materials. The light-cured set permits immediate placement and condensation of the restorative material. It contains approximately 45 percentage by weight (wt\%) mineral material (type III Portland cement), $10 \mathrm{wt} \%$ radiopaque component, $5 \mathrm{wt} \%$ hydrophilic thickening agent (fumed silica), and approximately $45 \mathrm{wt} \%$ resin [8]. In previous studies, TC showed good sealing abilities [8] and was well-tolerated by immortalized odontoblast cells [9]. It is necessary to evaluate whether that material has a mineral-inducing potential that can form a reparative dentin bridge. However, its mineral-inducing potential has not been studied yet.

This study aimed to evaluate the mineralization-inducing potentials of calcium silicate-based pulp capping materials on human dental pulp cells (HDPCs), by the following five outcome measures: (1) the amount of calcium release from the capping materials, (2) the $\mathrm{pH}$ values of aqueous medium exposed to the extracts of the capping materials which stimulate the pulp cell differentiation, (3) the cell viability by an MTT assay, (4) cytoskeletal organization of the HDPCs cultured on the extract of the capping materials and viewed with a microscope, and (5) alizarin red S staining images and alkaline phosphatase (ALP) activity of odontoblasts cultured on the capping materials.

\section{Materials and methods}

\section{Specimen preparation}

Premixed specimens of ProRoot MTA (PR; Dentsply, Tulsa, OK, USA), $\mathrm{BD}$, and TC were made according to the manufacturers' instructions (Table 1). Discs (10-mm diameter and 2-mm thickness) were made using a stainless-steel frame as a mold. Each disc was allowed to set for 24 hours followed by polishing with \#400, 600 , and 1,200 grit lapping film (3M lapping film; 3M, Maplewood, MN, USA). The colors of disc obtained by digital camera show the white color of MTA and TheraCal, and Biodentine has the Ivory color. The specimens were placed in a 48-well tissue culture plate, kept under an ultraviolet clean bench for 24 hours, and further sterilized by ultraviolet irradiation (Figs. 1, 2).

\section{Primary culture of human dental pulp cells}

HDPCs were provided by the Department of Oral Pathology (School of Dentistry, Kyungpook National University, Daegu, Korea). Fragments of pulp tissue acquired from an extracted human third molar were cultured in minimal essential medium alpha (MEM- $\alpha$; Invitrogen, Carlsbad, CA, USA) containing 10\% fetal bovine serum (FBS; Invitrogen), $100 \mathrm{U} / \mathrm{mL}$ penicillin, and 100 $\mathrm{U} / \mathrm{mL}$ streptomycin (Invitrogen). The cultures were stored in a

Table 1. Materials used in this study

\begin{tabular}{lr}
\hline Product & Concentration \\
\hline ProRoot MTA (Dentsply, Tulsa, OK, USA) & $75 \%$ \\
Portland cement & $20 \%$ \\
Bismuth oxide & $5 \%$ \\
Calcium sulfate & Packaged in capsule (0.7 g) \\
Biodentine (Septodont, Saint-Maur-des-Fosses, France) & Packaged in pipette (0.18 mL) \\
Powder: tricalcium silicate, dicalcium silicate, calcium carbonate, zirconium oxide, calcium oxide, iron oxide & $<60 \%$ \\
Liquid: calcium chloride, a hydrosoluble polymer, water & $<50 \%$ \\
TheraCal LC (Bisco Inc., Schamburg, IL, USA) & $<10 \%$ \\
Portland cement type III & $<$ \\
Polyethylene glycol dimethacrylate & $<$ \\
Barium zirconate & $<$ \\
\hline
\end{tabular}


humidified atmosphere of $5 \% \mathrm{CO}_{2}$ at $37^{\circ} \mathrm{C}$.

\section{Measurement of calcium release from pulp capping materials}

The calcium release of each specimen was measured to evaluate the mineralization-inducing potentials. The disc specimen was placed in $10 \mathrm{~mL}$ of deionized water for 1 and 7 hours and 1, 4, 7, 14, and 21 days. The amount of calcium ion released from the capping materials in the water was measured by inductively coupled plasma atomic emission spectroscopy (ICP; Optima 7300DV, PerkinElmer, Shelton, CT, USA). ICP is a technique for detecting trace metals. It excites atom and releases ions from electromagnetic radiation at characteristic wavelengths of a particular element.

\section{Measurement of $\mathrm{pH}$ value}

The $\mathrm{pH}$ values of each specimen were measured to assess the amount of eluted hydroxide ions, which stimulate the pulp cell differentiation. The disc specimen was placed in $10 \mathrm{~mL}$ of deion- ized water for 1 and 7 hours and 1, 4, 7, 14, and 21 days. The $\mathrm{pH}$ value of the aqueous medium, which was exposed to the extracts of the capping materials, was measured by a $\mathrm{pH}$ meter (Orion 3 Star, Thermo Scientific, Singapore). The $\mathrm{pH}$ apparatus was calibrated with solutions of $\mathrm{pH} 7.0$ and 4.0. The electrode was washed with ultrapure water and dried after every measurement.

\section{Cell viability test}

An MTT assay was conducted to evaluate the effects of the capping materials on cell viability. The amount of HDPCs on each specimen was measured by a colorimetric immune assay that is based on measuring bromodeoxyuridine (BrdU) incorporated during DNA synthesis. BrdU enzyme-linked immunosorbent assay (ELISA; Roche Molecular Biochemicals, Nutley, NJ, USA) was performed according to the manufacturer's instructions. Cells were seeded at a density of $2 \times 10^{4}$ cells per well onto a 48 -well plate and were cultured for 1 and 3 days with each specimen. Then, the BrdU-labeling solution was added to each well. The solution was applied to the cells in a $\mathrm{CO}_{2}$ incubator at $37^{\circ} \mathrm{C}$ for 4
A

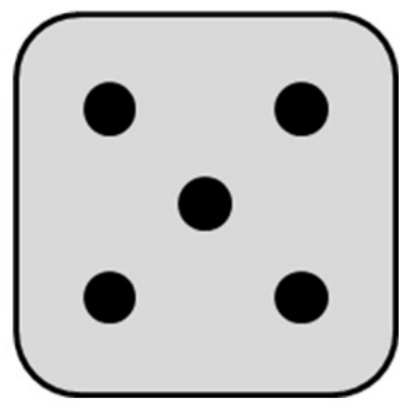

B

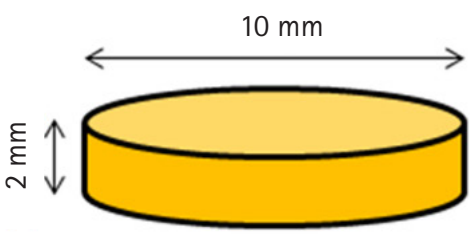

Fig. 1. (A) Stainless-steel frame with five holes, (B) schematic diagram of the specimen, (C) 48-well cell culture plates, having flat bottom which matches the specimen in size.
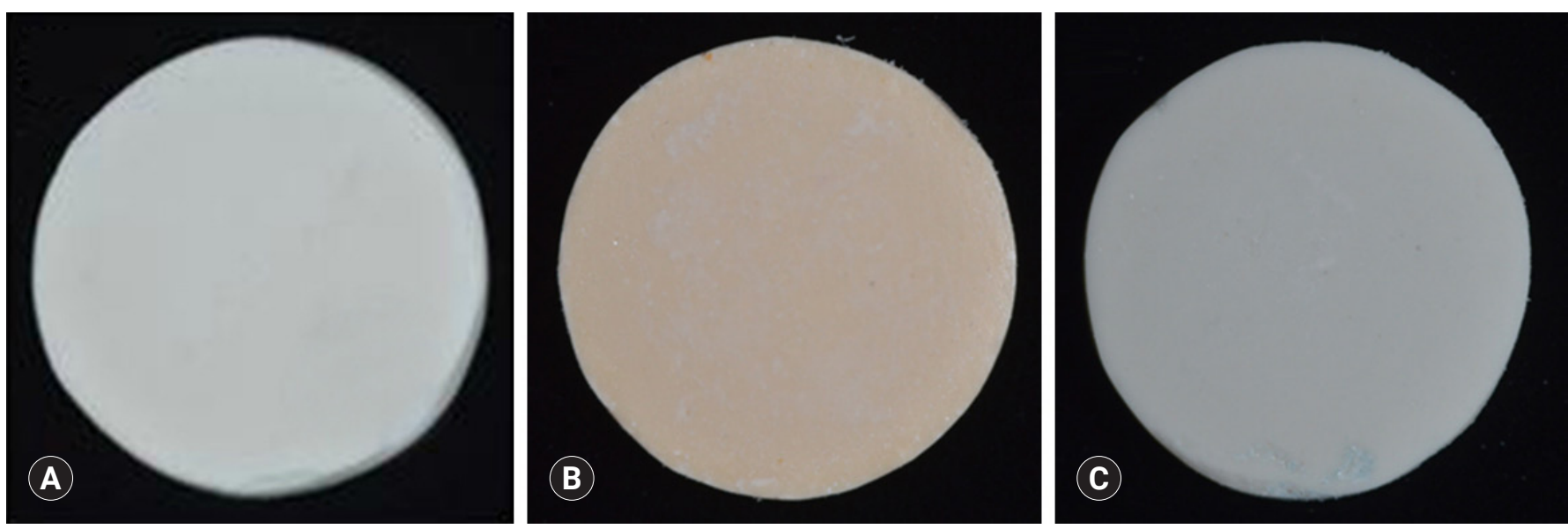

Fig. 2. Images of specimens. (A) ProRoot MTA, (B) Biodentine, (C) TheraCal LC. 
hours. The supernatant of the cell solution was removed by pipetting and were washed with phosphate-buffered saline (PBS) twice. After treating the cells with $0.25 \%$ trypsin-ethylenediaminetetraacetic acid (EDTA; Gibco, Tokyo, Japan), the cells were harvested by centrifugation at $1,000 \mathrm{rpm}$ for 15 minutes. For fixing and denaturing DNA, the harvested cells were mixed with FixDenat (ELISA; Roche Molecular Biochemicals) solution and then incubated for 30 minutes. Diluted anti-BrdU peroxidase (dilution ratio of 1:100) was added to the cells, which was then incubated at $20^{\circ} \mathrm{C}$ for 2 hours. The unbound antibody conjugate was removed, and $100 \mu \mathrm{L}$ substrate was added to the 48 -well plate. Then, the cells were incubated for 20 minutes. The reaction was finished by adding $25 \mu \mathrm{L}$ of $\mathrm{H}_{2} \mathrm{SO}_{4}$ solution $(1 \mathrm{M})$ to the cells. The solution was moved to a 96-well plate, and the absorbance of the solution was measured using an ELISA plate reader (EL 9800; Roche Molecular Biochemicals) for 5 minutes at $450 \mathrm{~nm}$ with a reference wavelength of $690 \mathrm{~nm}$. The blank reading corresponded to $100 \mu \mathrm{L}$ of culture medium with or without BrdU.

\section{Cytoskeletal organization}

To assess the cytoskeletal organization of HDPCs, which were treated with the extracts of the capping materials, double staining was performed. Briefly, a prepared specimen of each cement was stored in $10 \mathrm{~mL}$ MEM- $\alpha$ contained $10 \%$ FBS for 3 days to produce the extracts used for the treatment of HDPCs. The cells were seeded onto a 48 -well plate $\left(2 \times 10^{4}\right.$ cells $\left./ \mathrm{mL}\right)$ and were cultured for 1 day with the extracts of the specimen. The cells were fixed with $4 \%$ paraformaldehyde in PBS and washed with a PBS solution containing $0.05 \%$ Tween-20. After permeabilization with $0.1 \%$ Triton X-100 in PBS for 15 minutes at $25^{\circ} \mathrm{C}$, the cells were incubated for 30 minutes in PBS containing $1 \%$ bovine serum albumin. Subsequently, 5(6)- tetramethyl-rhodamine isothiocyanate-conjugated phalloidin (Millipore, Temecula, CA, USA) was added to the 48-well plate, and the cells were incubated for approximately 1 hour. The plates were washed three times (10 minutes each) using the buffer solution and incubated with 4',6-diamidino-2-phenylindole (Millipore) for 5 minutes. The scaffolds were washed three times (10 minutes each) with the buffer solution, and fluorescence images were visualized by a CLSM (model 700; Carl Zeiss, Oberkochen, Germany).

\section{Alizarin red S staining}

Mineralized nodules from differentiated cells were visualized through alizarin red S staining to evaluate the mineralization-inducing potentials of each specimen. Cells were seeded at a density of $2 \times 10^{4}$ cells per well onto a 48 -well plate and were cultured for 14 days with each specimen. Then, mineralization of the cells was evaluated through alizarin red S staining (Sigma-Aldrich, St. Louis, MO, USA). Briefly, cells were fixed with $4 \%$ formalin for 1 hour at $4 \%$, washed three times with deionized water, and then stained with $40 \mathrm{mmol} / \mathrm{L}$ of alizarin red solution $(\mathrm{pH}=4.2)$. The stained cell plate was washed with deionized water, and the stained image was obtained with the scanner. To remove the stain, the samples were treated with $10 \%$ cetylpyridinium chloride solution $(\mathrm{pH}=7.0)$ for $15 \mathrm{~min}$ utes, and absorbance was measured at a wavelength of $540 \mathrm{~nm}$ with a standard solution for the quantitative assessment.

\section{Alkaline phosphatase activity assay}

ALP is the marker of early differentiation and extracellular matrix mineralization. Cells were seeded at a density of $2 \times 10^{4}$ cells per well onto a 48-well plate and were cultured for 14 and 21 days with each specimen. Then, the cells were scraped into cold PBS and sonicated with a cell disruptor (Heat System Ultrasonics, Plainview, NJ, USA) in an ice-cold bath. ALP activity in the supernatant was determined using $\mathrm{p}$-nitrophenyl phosphate as a substrate. Absorbance was measured at $410 \mathrm{~nm}$ using an ELISA plate reader.

\section{Statistical analysis}

Statistical analysis of the data from calcium ion release, $\mathrm{pH}$, MTT assay, and ALP activity test was performed using IBM SPSS version 20.0 (IBM Corp., Armonk, NY, USA). One-way ANOVA, followed by the Tukey test, was performed. The level of significance was established at 0.05 .

\section{Results}

\section{Measurement of calcium release}

$\mathrm{BD}$ showed significantly higher calcium release compared to $\mathrm{PR}$ and TC in all test periods $(p<0.05)$ (Fig. 3). Calcium release from PR was significantly higher than TC $(p<0.05)$. The amount of calcium released from both $\mathrm{BD}$ and $\mathrm{PR}$ constantly increased with increased immersing time. However, the calcium release from TC slowly increased up to 4 days and thereafter almost stopped.

\section{Measurement of $\mathrm{pH}$ value}

After 1-day immersion, $\mathrm{PR}$ and $\mathrm{BD}$ had similar $\mathrm{pH}$ values, but TC showed lower $\mathrm{pH}$ value compared with others (Fig. 4). In the early stage, $\mathrm{PR}$ showed the highest $\mathrm{pH}$ value compared with others. All experimental groups showed high alkalinity near $\mathrm{pH} 11.0$ after 1 day, except at 14 days. There was no significant difference in $\mathrm{pH}$ values between $\mathrm{PR}, \mathrm{BD}$, and TC after 1 day, except at 14 days $(p>0.05)$. 


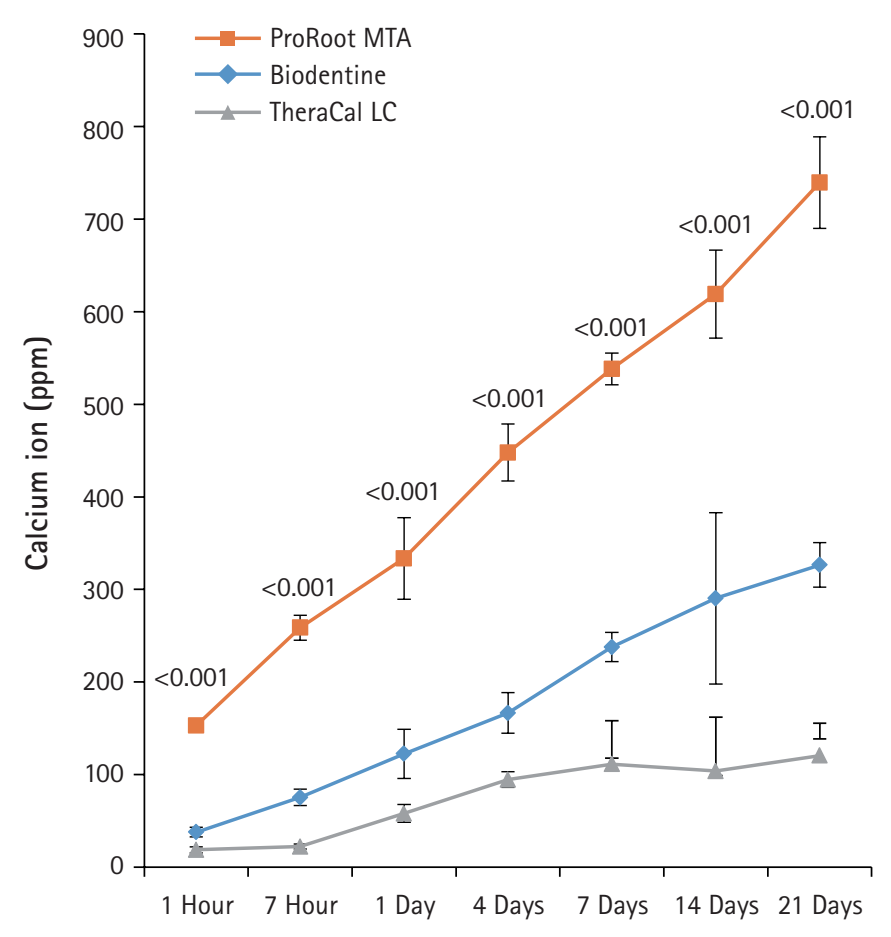

Fig. 3. The amount of calcium released from ProRoot MTA, Biodentine, and TheraCal LC in deionized water as a function of immersion time. The amount of calcium released from ProRoot MTA and Biodentine increased constantly with the immersion time, whereas the released calcium from TheraCal LC had a tendency to increase until day 4 , and almost stopped increasing. For each of the three types of samples, five samples were used at each time of the experiment. Error bars indicate standard errors of the means.

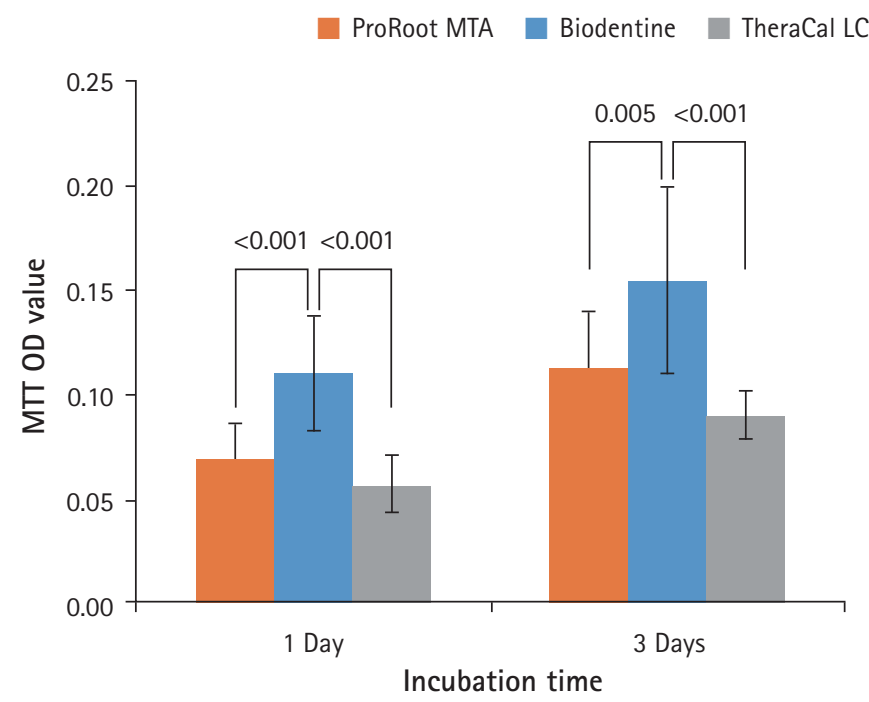

Fig. 5. Effects of ProRoot MTA, Biodentine, and TheraCal LC on cell viability measured by MTT assay. On both 1 and 3 days, the cell viability for Biodentine was highest. However, ProRoot MTA and TheraCal LC showed no significant difference $(p<0.05)$. For each of the three types of samples, six samples were used at each time of the experiment. Error bars indicate standard errors of the means. MTT OD value: cell viability absorbance $(490 \mathrm{~nm})$.

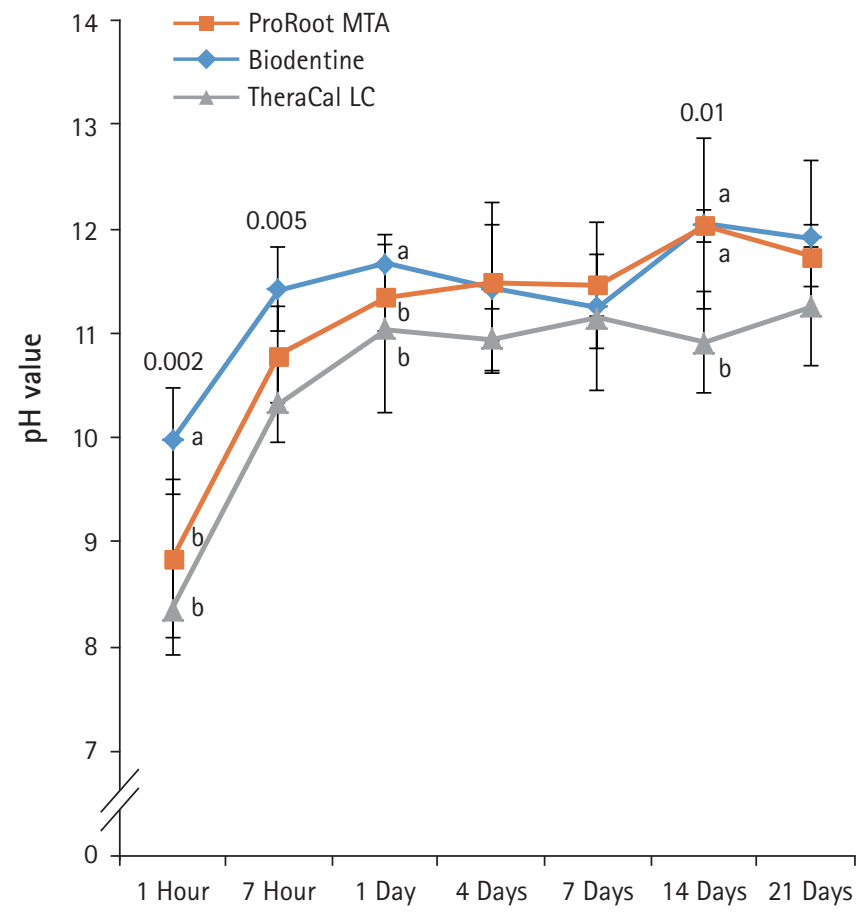

Fig. 4. pH values of aqueous medium exposed to the extracts of the capping materials as a function of immersion time. $\mathrm{pH}$ values for ProRoot MTA, Biodentine, and TheraCal LC were stable in near 11.0 after 1 day, except at 14 days. Values not sharing a common letter $(a, b)$ are significantly different $(p<0.05)$. For each of the three types of samples, five samples were used at each time of the experiment. Error bars indicate standard errors of the means.

\section{Cell viability test}

As shown in Fig. 5, BD showed the highest cell viability compared with PR and TC on both 1 day and 3 days. TC showed significantly lower cell viability than $\mathrm{BD}$, but there was no significant difference between PR and TC $(p<0.05)$.

\section{Cell morphologic analysis}

Cells were observed by confocal laser scanning microscopy to evaluate cell growth and morphology. As shown in Fig. 6, cells are well adhered in the shape of the fusiform and expressed actin filaments for all pulp capping materials.

\section{Alizarin red S staining and alkaline phosphatase activity test}

There was a high increase in mineralization in the PR and $\mathrm{BD}$ groups compared with the TC group based on alizarin red $S$ staining for calcium (Fig. 7). However, there was no difference in mineralization between PR and BD. In terms of ALP activity, there was a significant difference between the three groups. $\mathrm{BD}$ showed 

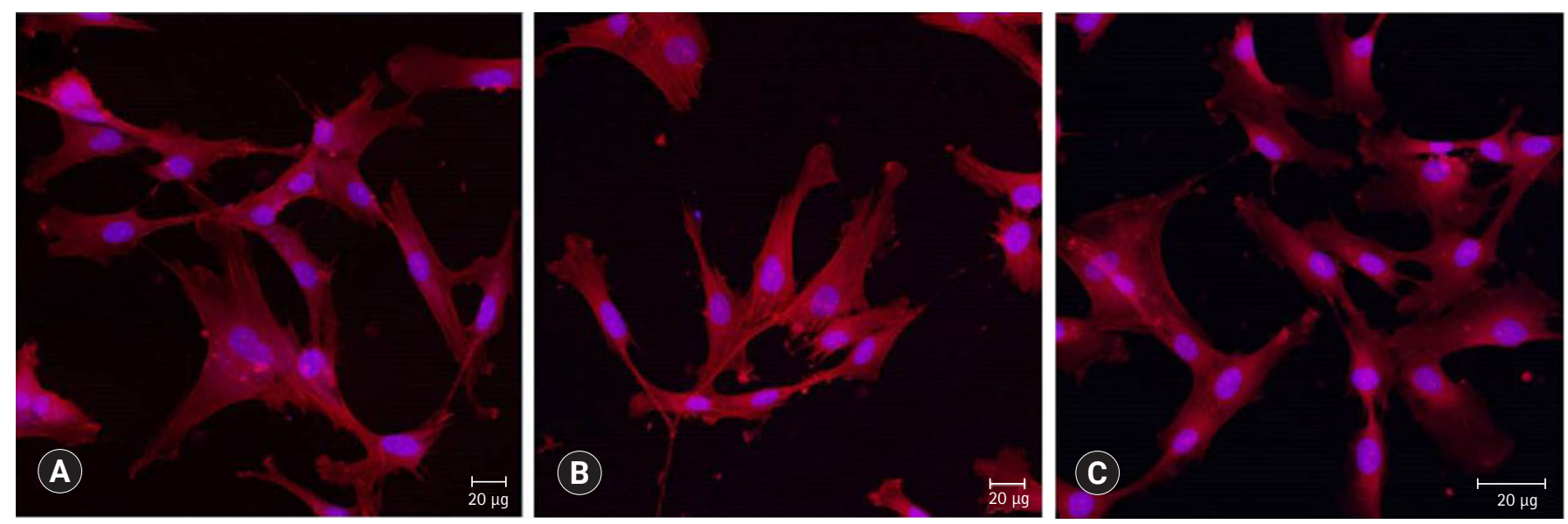

Fig. 6. Confocal laser scanning microscopic images (actin [red], nucleus [blue]) of HDPCs cultured on (A) ProRoot MTA, (B) Biodentine, and $(C)$ TheraCal LC which were incubated in the extract of the materials for 3 days. Cells were well adhered and expressed actin filaments for all pulp capping materials $(\times 200)$.
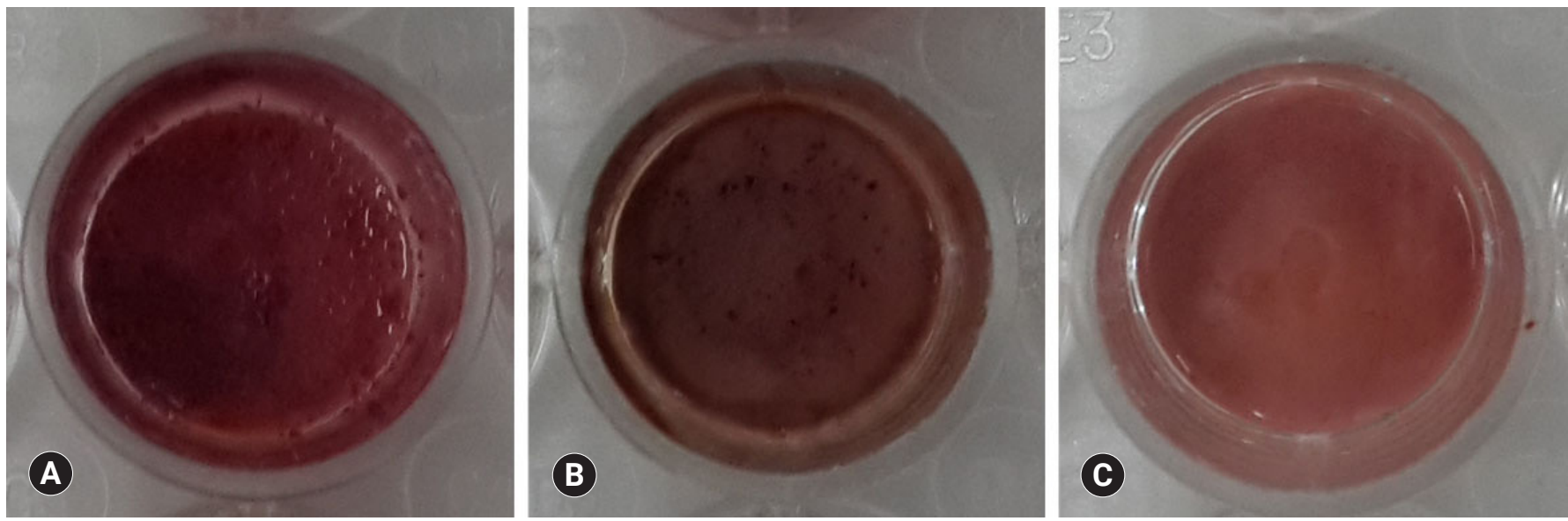

Fig. 7. Alizarin red S staining images of odontoblasts cultured on (A) ProRoot MTA, (B) Biodentine, and (C) TheraCal LC for 14 days. There was a high increase in mineralization in the ProRoot MTA and Biodentine groups compared with the TheraCal LC group based on alizarin red S staining for calcium.

a significantly higher value than $\mathrm{PR}$ and $\mathrm{TC}$, while TC showed the lowest activity compared to the others $(p<0.05)$ (Fig. 8).

\section{Discussion}

This study was designed to investigate the mineralization-inducing potentials of pulp capping materials on cultured HDPCs.

In regards to the calcium ion release, BD showed a markedly higher release of free calcium ions compared with PR and TC. The increased calcium release from $\mathrm{BD}$ has been considered to be correlated with the presence of a calcium silicate component, calcium chloride, and low solubility (11.93\%). This is likely linked to a superplasticizer that is commonly used to reduce the amount of water required (L/P 0.257) to disperse the particles and to enhance the fluidity, making the cement self-consolidating. In addition, the fast hydration reaction of tricalcium silicate can be correlated with the low solubility and high calcium release at early endpoints [8]. In another previous study, BD exhibited advanced hydration both in vitro and after pulp capping. This was evident from the densely hydrated product in the cement matrix and the absence of unhydrated cement particles after 14 days of hydration [10].

In contrast, TC exhibited the lowest calcium ion leaching. This result can be attributed to the fact that the release of calcium ions is limited due to the presence of a resin-modified matrix in the structure of TC after setting. The restriction of fluid absorption 


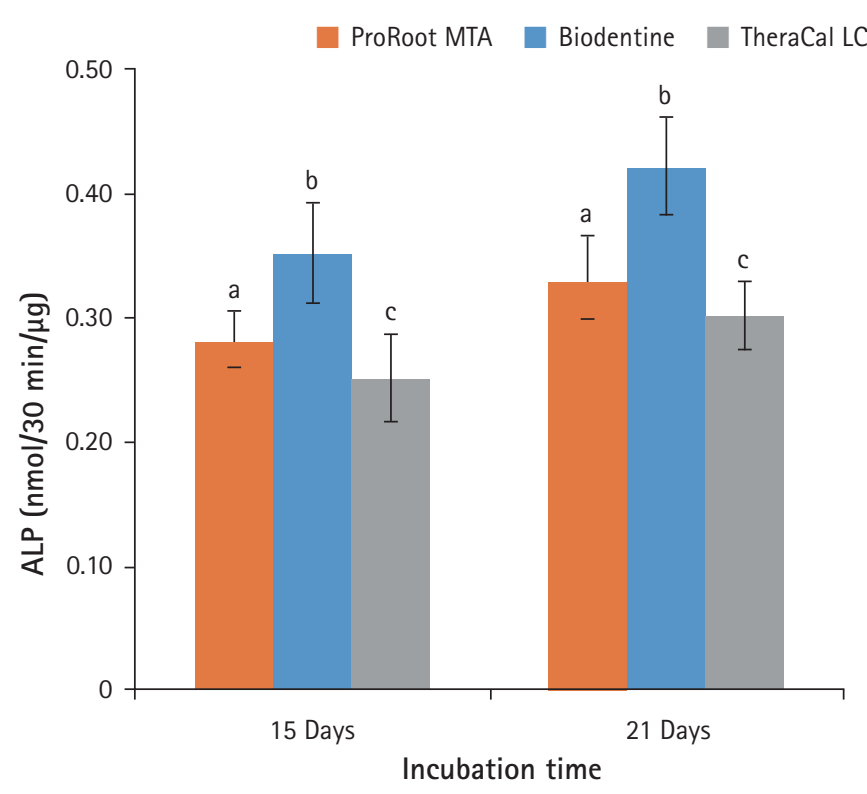

Fig. 8. Alkaline phosphatase (ALP) activity of odontoblasts cultured on pulp capping materials for 14 and 21 days. The ALP activity of odontoblasts in Biodentine group was highest on both observation days. However, the odontblasts in TheraCal LC group showed the lowest ALP activity $(p<0.05)$. For each of the three types of samples, five samples were used at each time of the experiment. Values not sharing a common letter $(a, b, c)$ are significantly different $(p<0.05)$. Error bars indicate standard errors of the means.

causes calcium ions to scarcely move along the resin matrix [10].

The release rate of calcium ions is a key factor for successful endodontic and pulp capping therapies because the mineralization of cells (osteoblasts, cementoblasts, pulp cells, and odontoblasts) are influenced by calcium ions [7]. Calcium ions specifically modulate osteopontin and bone morphogenetic protein- 2 levels during pulp calcification [11]. In addition, eluted calcium ions increase the proliferation of HDPCs in a dose-dependent manner [12], and calcium release enhances the activity of pyrophosphatase, which helps to maintain dentine mineralization and the formation of a dentin bridge [13].

To assess the amount of eluted hydroxide ions from the pulp capping materials, $\mathrm{pH}$ values of the aqueous medium, which was exposed to the extracts of the test materials, were measured. All materials showed similar alkaline activity. The $\mathrm{pH}$ values of all the test material reached a maximum of 11 after 7 hours, showing a plateau since 1 day immersion. This is in accordance with the results of previous studies $[14,15]$.

The high alkalinity of calcium hydroxide seems to result in mild stimulation of cell differentiation. Hydroxide ions stimulate the release of ALP and bone morphogenetic protein 2, which partici- pate in the mineralization process $[16,17]$.

The release of calcium hydroxide is mainly the result of the stimulation of odontoblast activity and subsequent mineralization. The pulp capping materials that are based on tricalcium silicate all allegedly release calcium hydroxide as a by-product of hydration. This has been demonstrated for MTA [18] and BD [19]. Calcium, as well as hydroxyl ions released from the capping materials, regulate the event leading to tertiary dentinogenesis. For the biological effects of calcium hydroxide, the release of bioactive molecules, either through direct stimulation of cells or by solubilization of dentin extracellular matrix, is vital. Calcium silicate cement, together with microcrystals deposited on its surface, provides a biologically active substrate for the adsorption of biomolecules and adhesion of odontoblasts [2].

In this study, the number of calcium ions released from the capping materials and $\mathrm{pH}$ value of the medium were measured in deionized water rather than simulated body fluid in order to standardize the test conditions and hence allow a comparison of the data with other future studies.

Prior to investigating the mineralization-inducing potentials of the materials, the biocompatibility was compared by evaluating the effects of the capping materials on cell morphology and cell viability in this study. BD showed better cell viability than PR and TC on the MTT assay. The result of the current study corresponded with an investigation by Poggio et al. [20], which showed BD induced a more favorable cell response due to high mitochondrial activity compared with MTA and TC during the 72 hours of incubation. Meanwhile, other researchers reported that $\mathrm{BD}$ showed similar cell viability to MTA $[5,21]$.

Confocal laser scanning microscopic images were used to compare cell morphology and cytoskeletal organization. When HDPCs were cultured on the capping materials for 1 day, the cultured cells appeared flat and showed well-defined cytoplasmic extensions, indicating all materials allowed cell attachment and growth. The actin microfilament cytoskeleton is involved in cell processes, cell shape, and cell attachment. As the cell adheres to a substrate material, filopodia are formed. They are moved into place by actin acting upon the plasma membrane. The actin is observed in the filopodia as directed tight parallel bundles. Contractile stress fibers are seen once the filopodia are attached [22]. Our results showed that the cytoskeletal organization of cells was observed, as shown in Fig. 6. The phenotype of differentiation-induced HDPCs is known to resemble several crucial characteristics of odontoblasts, such as increased ALP activity, mRNA expression of differentiation markers genes, and the formation of mineralized matrix in vitro [23].

ALP is the most frequently used marker of odontoblastic cell 
differentiation because ALP activity is greatest just before cell mineralization begins. In this study, odontoblastic differentiation of HDPCs was evaluated using an ALP activity assay. There was a significant increase in the ALP activity of the cells for BD, followed by PR and TC. This result is consistent with that of a previous study reporting the up-regulation of osteogenic or odontogenic genes by BD in HDPCs [24]. BD and PR promoted the odontoblastic differentiation with the development of mineralized nodules, which is the nucleation of the calcium phosphate layer on the surface of the material, whereas nodules were rarely observed in TC. Nowicka et al. [25] reported that the reparative tissues induced by two materials were homogeneous with the $\mathrm{BD}$ group, showing the dentin bridge of better quality. It was also reported that $\mathrm{BD}$ exerted significantly higher stimulatory activity on pulp cells in comparison with MTA after direct pulp capping to animal teeth, resulting in thicker reparative dentin bridges and greater incidence of ectopic pulp calcification in developing teeth [26].

In this study, TC showed relatively low odontoblastic differentiation compared with PR and BD. This result could be explained by several reasons. First, TC may undergo a complicated setting reaction by setting light-cured resin initially and hydrating tricalcium silicate, thereafter [27], inducing insufficient hydration. Moreover, TC is a resin-modified capping material, and it does not use water as mixing fluid. According to a previous study conducted by Camilleri et al. [10], the hydration of TC depends on fluid uptake through the resin matrix from the environment. Although there is calcium ion release from hydrating TC, no calcium hydroxide is formed, as has been verified by X-ray diffraction analysis in previous research. Instead, calcium phosphate was deposited over the TC surface [19]. Further study may be needed to reveal the precise setting mechanism of TC.

Next, cytotoxicity of the unpolymerized monomers could cause adverse effects on cellular metabolism [28]. Poggio et al. [20] reported that TC had slight cytotoxicity in comparison with $\mathrm{BD}$ and MTA-Angelus, which showed no cytotoxicity. Some resin monomers, especially 2-hydroxyethyl methacrylate (HEMA) and triethylene glycol dimethacrylate (TEGDMA), are commonly identified in eluates of polymerized composite resins [29]. According to Kwon et al. [30], the ALP activity of HDPCs was reduced by TEGDMA and HEMA at noncytotoxic concentrations. The mRNA expression of dentin sialophosphoprotein, osteocalcin, and osteopontin was also downregulated by resin monomers. Therefore, exposure to TEGDMA and HEMA for certain period leads to the suppression of the differentiation of HDPCs via different signaling pathways. Since TC is known to contain HEMA and TEGDMA, the degree of conversion and the amount of unpolymerized monomers of TC after light curing need to be investigated in further studies.

A limitation of this study is the lack of previous studies analyzing the precise setting mechanism of TC and the biological effects of TC. Although our results are promising, further studies are required to analyze the influence of other factors such as gene expression analysis by real-time polymerase chain reaction and protein expression analysis by western blotting. Furthermore, research on the effects of the immunological system and pulp tissue in vivo is also required.

Within the limitations of this in vitro study, it was found that $\mathrm{BD}$ had higher mineralization-inducing potentials than PR and $\mathrm{TC}$; therefore, $\mathrm{BD}$ is potentially suitable for use as a pulp capping material. TC showed similar biocompatibility to PR, while less effective on mineralization-inducing potentials.

\section{Acknowledgments}

\section{Conflicts of interest}

No potential conflict of interest relevant to this article was reported.

\section{Notes}

This paper is based on the author's doctoral dissertation (The Graduate School of Kyungpook National University, 2016).

\section{ORCID}

Sohee Kang, https://orcid.org/0000-0002-3667-1952

\section{References}

1. Smith AJ. Formation and repair of dentin in the adult. In: Hargreaves KM, Goodis HE, Tay FR, editors. Seltzer and Bender's dental pulp. 2nd ed. Hanover Park (IL): Quintessence Publishing; 2012.p. 27-46.

2. Sangwan P, Sangwan A, Duhan J, Rohilla A. Tertiary dentinogenesis with calcium hydroxide: a review of proposed mechanisms. Int Endod J 2013;46:3-19.

3. Okiji T, Yoshiba K. Reparative dentinogenesis induced by mineral trioxide aggregate: a review from the biological and physicochemical points of view. Int J Dent 2009;2009:464280.

4. Mente J, Geletneky B, Ohle M, Koch MJ, Friedrich Ding PG, Wolff $\mathrm{D}$, et al. Mineral trioxide aggregate or calcium hydroxide direct pulp capping: an analysis of the clinical treatment outcome. J Endod 2010;36:806-13.

5. Bogen G, Kim JS, Bakland LK. Direct pulp capping with mineral trioxide aggregate: an observational study. J Am Dent Assoc 2008;139:305-15. 
6. Chang SW, Lee SY, Ann HJ, Kum KY, Kim EC. Effects of calcium silicate endodontic cements on biocompatibility and mineralization-inducing potentials in human dental pulp cells. J Endod 2014;40:1194-200.

7. Gandolfi MG, Siboni F, Polimeni A, Bossu M, Riccitiello F, Rengo S, et al. In vitro screening of the apatite-forming ability, biointeractivity and physical properties of a tricalcium silicate material for endodontics and restorative dentistry. Dent J 2013; $1: 41-60$.

8. Suh B, Cannon M, Yin R, Martin DE, inventors. Polymerizable dental pulp healing, capping, and lining material and method for use. International patent A61K33/42. 2008 Aug 28.

9. Hebling J, Lessa FC, Nogueira I, Carvalho RM, Costa CA. Cytotoxicity of resin-based light-cured liners. Am J Dent 2009; 22:137-42.

10. Camilleri J, Laurent P, About I. Hydration of Biodentine, Theracal LC, and a prototype tricalcium silicate-based dentin replacement material after pulp capping in entire tooth cultures. J En$\operatorname{dod} 2014 ; 40: 1846-54$.

11. Rashid F, Shiba H, Mizuno N, Mouri Y, Fujita T, Shinohara H, et al. The effect of extracellular calcium ion on gene expression of bone-related proteins in human pulp cells. J Endod 2003;29:104-7.

12. Takita T, Hayashi M, Takeichi O, Ogiso B, Suzuki N, Otsuka K, et al. Effect of mineral trioxide aggregate on proliferation of cultured human dental pulp cells. Int Endod J 2006;39:415-22.

13. Estrela C, Holland R. Calcium hydroxide: study based on scientific evidences. J Appl Oral Sci 2003;11:269-82.

14. Formosa LM, Mallia B, Camilleri J. The chemical properties of light- and chemical-curing composites with mineral trioxide aggregate filler. Dent Mater 2013;29:e11-9.

15. Grech L, Mallia B, Camilleri J. Characterization of set Intermediate Restorative Material, Biodentine, Bioaggregate and a prototype calcium silicate cement for use as root-end filling materials. Int Endod J 2013;46:632-41.

16. Moghaddame-Jafari S, Mantellini MG, Botero TM, McDonald NJ, Nor JE. Effect of ProRoot MTA on pulp cell apoptosis and proliferation in vitro. J Endod 2005;31:387-91.

17. Maeda H, Nakano T, Tomokiyo A, Fujii S, Wada N, Monnouchi $\mathrm{S}$, et al. Mineral trioxide aggregate induces bone morphogenetic protein-2 expression and calcification in human periodontal ligament cells. J Endod 2010;36:647-52.

18. Camilleri J. Characterization of hydration products of mineral trioxide aggregate. Int Endod J 2008;41:408-17.

19. Camilleri J. Hydration characteristics of Biodentine and Theracal used as pulp capping materials. Dent Mater 2014;30:70915.

20. Poggio C, Arciola CR, Beltrami R, Monaco A, Dagna A, Lombardini $\mathrm{M}$, et al. Cytocompatibility and antibacterial properties of capping materials. ScientificWorldJournal 2014;2014: 181945.

21. Corral Nunez CM, Bosomworth HJ, Field C, Whitworth JM, Valentine RA. Biodentine and mineral trioxide aggregate induce similar cellular responses in a fibroblast cell line. J Endod 2014;40:406-11.

22. Burridge K, Chrzanowska-Wodnicka M. Focal adhesions, contractility, and signaling. Annu Rev Cell Dev Biol 1996;12:463518.

23. Gronthos S, Mankani M, Brahim J, Robey PG, Shi S. Postnatal human dental pulp stem cells (DPSCs) in vitro and in vivo. Proc Natl Acad Sci U S A 2000;97:13625-30.

24. Laurent P, Camps J, About I. Biodentine(TM) induces TGF- $\beta 1$ release from human pulp cells and early dental pulp mineralization. Int Endod J 2012;45:439-48.

25. Nowicka A, Wilk G, Lipski M, Kolecki J, Buczkowska-Radlinska J. Tomographic evaluation of reparative dentin formation after direct pulp capping with $\mathrm{Ca}(\mathrm{OH}) 2$, MTA, Biodentine, and dentin bonding system in human teeth. J Endod 2015;41: 1234-40.

26. Tziafa C, Koliniotou-Koumpia E, Papadimitriou S, Tziafas D. Dentinogenic responses after direct pulp capping of miniature swine teeth with Biodentine. J Endod 2014;40:1967-71.

27. Chung H, Kim M, Ko H, Yang W. Evaluation of physical and biologic properties of the mixture of mineral trioxide aggregate and 4-META/MMA-TBB resin. Oral Surg Oral Med Oral Pathol Oral Radiol Endod 2011;112:e6-11.

28. Hanks CT, Strawn SE, Wataha JC, Craig RG. Cytotoxic effects of resin components on cultured mammalian fibroblasts.J Dent Res 1991;70:1450-5.

29. Michelsen VB, Lygre H, Skalevik R, Tveit AB, Solheim E. Identification of organic eluates from four polymer-based dental filling materials. Eur J Oral Sci 2003;111:263-71.

30. Kwon JH, Park HC, Zhu T, Yang HC. Inhibition of odontogenic differentiation of human dental pulp cells by dental resin monomers. Biomater Res 2015;19:8. 